\title{
Uncomputable but complete physics theory of the universe
}

\author{
William Icefield \\ (Dated: October 10, 2020)
}

\begin{abstract}
It is often assumed that the complete physics theory of the universe is computable - in sense that it can provide meaningful theoretical predictions for every phenomenon of the universe. Against this view, it is argued that once quantum mechanics is understood as encompassing a novel concept of probability, thereby resolving the measurement problem in straightforward ways, the interpretation speaks for uncomputability of the complete physics theory. The reasons why a new theory of probability is needed are explored, along the lines of the principle of indifference and the sleeping beauty problem.
\end{abstract}

Keywords: uncomputable theory, generalized probability, measurement problem, principle of indifference, Sleeping Beauty Problem 


\section{CONTENTS}

$\begin{array}{ll}\text { I. Introduction } & 2\end{array}$

II. Measurement problem: breakdown of classical probability 3

A. Textbook view and statistical alternative 4

B. Remaining subjectivity issue? Uncomputable complete theory 5

III. Issues with classical probability and the alternative 6

A. A problem with the principle of indifference $\quad 6$

$\begin{array}{ll}\text { B. Sleeping Beauty Problem } & 7\end{array}$

IV. Conclusion $\quad 8$

$\begin{array}{ll}\text { References } & 9\end{array}$

\section{INTRODUCTION}

This paper first explores the measurement problem as suggesting a novel concept of probability, with breakdown of the classical probability theory arising out of Kolmogorov axioms. What probability theory is required by quantum mechanics is explored. The new probability theory implies that the full theory of the universe is uncomputable - it cannot be derived or used for predictions.

The key issue behind the measurement problem is that measurements affect probability in a very non-trivial way, so how we use the usual classical probability theory needs to be modified in order to make sense of reality. One question is whether this is because reality is not intuitive or because the concept of probability needs to be changed - here, we take the side of the need to change the concept of probability.

Assume again that the state of the universe can be imagined even hypothetically and theoretically. But given that Equation (1) breaks down in quantum mechanics - and since the state of the universe being entirely known is theoretically equivalent to having measured the universe in the 'novel probability' view of quantum mechanics - this would break quantum mechanics itself, because the circumstance is where the classical probability theory should apply. (Equation (1) in this circumstance means initially assigning probability to orthogonal 
universe outcomes at time $t$ and calculating probability of orthogonal universe outcomes at time $t^{\prime} \geq t$.) Therefore in this view, the full theory of the universe is uncomputable. Illusions of subjectivity in the measurement problem come from this issue.

We further explore why a novel probability theory would be required, along the lines of the principle of indifference[1] and the sleeping beauty problem[2-4].

\section{MEASUREMENT PROBLEM: BREAKDOWN OF CLASSICAL PROBABIL- ITY}

In the conventional and classical theory of probability that comes from Kolmogorov axioms, the following equation then holds:

$$
\sum_{i_{0} \in I_{t_{0}}} P\left(i_{0}\right) P\left(f_{1} \mid i_{0}\right)=P\left(f_{1}\right)
$$

$I_{t_{0}}$ refers to a set of all possible mutually orthogonal outcomes at time $t_{0}, f_{1} \in I_{t_{1}}$ refers to one specific outcome at time $t_{1} \cdot P(\cdot)$ refers to (unconditional) probability measure, with $P(\cdot \mid \cdot)$ referring to probability measure conditional on the latter term after $\mid . t_{1} \geq t_{0}$.

In quantum mechanics, Equation (1) breaks down - that is, the equation does not hold all the time. This is the most important issue revolving around the measurement problem. (The other issue is about a preferred measurement basis, which would be discussed together given that it is actually the same issue in the 'novel probability' view of quantum mechanics.) [5] In essence, Equation (1) is what surrounds Bell's theorem[6], Bell-Kochen-Specker theorem[7, 8], their variants and Hardy's paradox[9] as well.

From the probability theory perspective, unless we have reasons to stick with the classical probability theory, it is unclear why breakdown of Equation (1) means wavefunction collapse. Maybe, reality could be asking for the use of an alternative theory of probability. Breakdown of the classical probability theory may open up for the new type of statistical correlation that quantum mechanics requires, as visible in Bell-type inequalities. Instead of explaining quantum correlations in terms of (non-)existence of (local) reality, we could instead think of them in terms of a new generalized probability theory and the corresponding generalized theory of correlations. In fact, quantum reconstruction projects have provided rationality criteria that can derive quantum mechanics.[10-12]

In the classical probability theory, measurements themselves do not necessarily affect 
probability arithmetic. When calculating $P\left(f_{1}\right)$ in the classical probability theory, one does not have to consider whether particular outcome $i_{0}$ was measured at time $t_{0}$. Because one does not hold knowledge of what outcome $i_{0}$ held in reality, one can simply proceed to use unconditional probability (relative to $\left.I_{0}\right) P\left(f_{1}\right)$ as genuine probability measure of outcome $f_{1}$.

In quantum mechanics, because of breakdown of Equation (1), it matters whether an outcome was measured in time $t_{0}$. If Agent $A$ measured particular outcome $i_{0}$, then even if agent $B$ does not recognize measurement and observation results of agent $A$, unconditional $P\left(f_{1}\right)$ would no longer be valid probability measure of outcome $f_{1}$. That is, $P\left(f_{1}\right)$ in such a case has to be calculated in quantum mechanics as $P\left(f_{1}\right)=P\left(f_{1} \mid i_{0}\right)$. In fact, this feature is used in quantum cryptography (for communication) to check presence of an eavesdropper.[13]

\section{A. Textbook view and statistical alternative}

Quantum states are largely thought to evolve continuously via the Schrödinger equation before a measurement. Thus, the conventional textbook view has been that state (almost identically, wavefunction) collapse occurred when measurements are done.

The textbook view often is stated in terms of the anti-realism view: reality simply does not exist before measurements. That is why Equation (1) breaks down - probability arithmetic only makes sense for potentially realizable outcomes - and realizable outcomes are determined when measurements are conducted. This raises the question of why measurement decisions matter - it seems to introduce subjective aspects into physics.

If we think of arithmetic and rules of quantum mechanics as a generalized probability theory that complements, generalizes and, as to be visited, repairs the classical probability theory that follows from Kolmogorov axioms, then the issue regarding realism versus antirealism is simply dissolved. Because agents already possess knowledge that the generalized probability theory in use allows for measurements to significantly interfere with probability arithmetic, allowing for breakdown of Equation (1), there is no surprise. As with the quantum cryptography example, if an agent notices that an experiment result does not match with expected probability, this simply means that the agent does not have full knowledge (or model) of what is going on. In case of the quantum cryptography example, Agent $A$ and $B$ conduct an experiment, expecting non-existence of an eavesdropper - if an actual result 
deviates from an expected result, that would mean that a previously unnoticed (from the statistical model) eavesdropper exists.

The whole circumstance is similar to what happens in statistics - a statistician has a statistical model of reality and experiments with reality - if the result suggests reality deviating from the model, then the model is repaired.

\section{B. Remaining subjectivity issue? Uncomputable complete theory}

One may argue that this still leaves subjectivity issues - it is not simply enough for agents to know how measurements proceeded. What measurement outcome was actually observed plays a significant role in probability arithmetic of quantum mechanics. For example, if an eavesdropper exists while Agent $A$ and $B$ are conducting quantum communication experiments, then to obtain valid probability measure at each time moment, what the eavesdropper measured - not just the fact that the eavesdropper exists - is important.

Does this really mean that subjectivity plays a significant role in quantum mechanics? No - in fact, there was a misunderstanding in the aforementioned statement. To understand this point, we need to think of what the new probability theory presented by quantum mechanics - which we may call as the quantum probability theory - says. Again, the argument is replicated. Suppose that we can even imagine the state of the full universe. But in such a case, this would be theoretically equivalent to having measured the entire universe. Furthermore, Equation (1) should hold for the entire universe: by Equation (1) for the entire universe, we are saying that probability is assigned for each possible orthogonal universe outcome at time $t$, and then we ask probability of each possible orthogonal universe outcome at time $t^{\prime} \geq t$. In this circumstance, because each state of the universe is 'the' state of the universe, one cannot imagine orthogonal states interfering with each other in the present or future. Yet quantum mechanics suggests otherwise. Thus, the complete theory of the universe must then be uncomputable - it cannot be derived or used. (An alternative view of quantum mechanics, given by the Everettian many-worlds view, may be suggested[14] - we would not discuss this view furthermore, other than this brief mention. There, different worlds and universes may interfere with each other.)

Therefore, all we can do is to note what measurement procedures are experimentally confirmed to be reliable and to use these procedures to explore and update theories that 
may provide new measurement procedures. Using these existing measurement procedures, we note down available measurement outcomes and check against existing theories and models.

It is not subjective decisions per se that drive the measurement problem under the probability theory view of quantum mechanics - it is the limitation (that the complete theory of the universe is uncomputable) induced by quantum mechanics that creates illusions of subjectivity and the measurement problem. While the objective universe exists, we cannot fully access it, leaving appearance of subjectivity.

\section{ISSUES WITH CLASSICAL PROBABILITY AND THE ALTERNATIVE}

There already were several issues with the classical probability theory that call for a new theory of probability. Two issues are explored: the issue revolving around the principle of indifference and the sleeping beauty problem.

\section{A. A problem with the principle of indifference}

The problem is simple. Suppose we know that random variable $X$ has support on $(0,1)$ but we know nothing else. If we invoke the principle of indifference, then we would assign $P(x)=1$ for $0<x<1$. However, when we think of random variable $Y$ with $y=x^{2}$, then $Y$ also has support on $(0,1)$. Furthermore, we also have no other knowledge for $Y$ as well. Invoking the principle of indifference to $Y$ suggests that $P(y)=1$ for $0<y<1$. Yet this is incompatible with the principle of indifference applied to $X$. Bertrand's paradox can be considered to be a more sophisticated variant of this issue[1] as well.

The quantum probability theory resolves this issue, which can be checked from the fact that the fundamental measure of quantum uncertainty is von Neumann entropy. There, von Neumann entropy is unaffected by linear (basis) transformations, in contrast to Shannon

entropy in the classical probability theory. Surely, in some sense, this really is brushing aside the issue. We are still allowing for multiple unitarily inequivalent representations that essentially encode the same 'theory.' Yet the reason why we have the issue with the principle of indifference (or the principle of maximum entropy, in general) becomes much more mathematically clearer - the quantum probability theory allows for multiple unitarily 
inequivalent representations, which force us to make some choice beforehand.

\section{B. Sleeping Beauty Problem}

There is and will likely be no unique consensus to the Sleeping Beauty problem. Here, it would be assumed that the thirder resolution $[3,4]$ is correct.

The thirder argument presents a challenge against the classical probability theory - but before continuing on, the Sleeping Beauty problem would briefly be explained. On Sunday, a fair coin is tossed. Sleeping Beauty possesses no knowledge of the coin toss outcome and is put to sleep. If head comes up, then Sleeping Beauty is awakened and interviewed only on Monday. If tail comes up, then Sleeping Beauty is awakened and interviewed twice - on Monday and Tuesday. For both coin toss outcomes, after the Monday interview is finished, Sleeping Beauty is put back to sleep. In each interview, Sleeping Beauty is asked: what credence does Sleeping Beauty assign on each coin toss outcome? The thirder position argues that $P($ head $)=1 / 3$, while the halfer position argues that $P($ head $)=1 / 2$.

The thirder argument is in conflict with the classical probability view that because $P($ head $)=1 / 2$ unconditionally on Sunday and no new knowledge has been provided to Sleeping Beauty, $P($ head $)=1 / 2$ should be maintained. The original and canonical thirder argument by Adam Elga[3] invokes the principle of indifference - therefore, it is possible to view that even the Sleeping Beauty problem is a variant of the general principle of indifference issue. Here, the additional issue is that one justifiable way of invoking the principle of indifference seems simply incompatible with the classical probability theory.

The canonical response to this issue is that credence assignment is to be distinguished from probability assignment. However, it is unclear how we should separate credence from probability - this involving the complicated question of what probability actually refers to. It may be argued that objective probability exists separately from subjective experience of

probability that we may call as credence, but then we have to reconsider how subjective Bayesian notions of probability may be reconciled with the classical probability theory. We do not experience objective probability - we experience subjective probability. If so, then what would be the point about separating credence from probability?

Thus, it is more reasonable to justify the thirder argument in terms of a new generalized probability theory, rather than sticking with the classical probability theory. In this view, 
the Sleeping Beauty problem shows that a new theory of probability is needed. The quantum probability theory provides why $P$ (head) may be $1 / 2$ at some moment, while $1 / 3$ at some other moments. Let us again remind of breakdown of Equation (1), but this time with time reversed:

$$
\sum_{i_{1} \in I_{t_{1}}} P\left(i_{1}\right) P\left(f_{0} \mid i_{1}\right) \neq P\left(f_{0}\right)
$$

Or restate as to align with the Sleeping Beauty problem:

$$
P(\text { Mon }) P(\text { head } \mid \text { Mon })+P(\text { Tue }) P(\text { head } \mid \text { Tue }) \neq P(\text { head })
$$

where Mon refers to Monday, Tue refers to Tuesday.

\section{CONCLUSION}

One important point of this paper is to establish that myths, mysteries and controversies around quantum mechanics disappear when we consider it as a generalized probability theory that we may call as the quantum probability theory. Rules and arithmetic of quantum mechanics are to be viewed as probabilistic principles. The measurement problem seems to arise because we are clinging onto arithmetic of the classical probability theory, represented by Equation (1). The purported subjective observer requirement for quantum mechanics is an illusion - it only arises because under the new probability theory, the state of the entire universe cannot even be theoretically imagined - otherwise, inconsistency arises. This means that the complete physics theory of the universe is uncomputable - we cannot derive or use it. This puts limitations on our knowledge of the universe, which seems to open up for elements of subjectivity, when they are just illusions.

The question could be raised - why is a new probability theory necessary? The discussions around the principle of indifference[1] and the Sleeping Beauty Problem[2-4] addressed this question - indeed, the quantum probability theory is necessary. The question of deriving the quantum probability theory was relegated to quantum reconstruction projects, which derive

quantum mechanics from epistemic requirements[10-12]. Once epistemic conditions require that only the classical probability theory and the quantum probability theory are possible, 
we are done in firmly establishing quantum mechanics as a necessary probability theory.

[1] Alon Drory, "Failure and Uses of Jaynes' Principle of Transformation Groups," Foundations of Physics 45, 439-460 (2015).

[2] Arnold Zuboff, "One self: The logic of experience," Inquiry 33, 39-68 (1990), https://doi.org/10.1080/00201749008602210.

[3] Adam Elga, "Self-locating belief and the Sleeping Beauty problem," Analysis 60, 143-147 (2000), https://academic.oup.com/analysis/article-pdf/60/2/143/466877/60-2-143.pdf.

[4] Berry Groisman, Na'ama Hallakoun, and Lev Vaidman, "The measure of existence of a quantum world and the Sleeping Beauty Problem," Analysis 73, 695-706 (2013).

[5] Maximilian Schlosshauer, "Decoherence, the measurement problem, and interpretations of quantum mechanics," Rev. Mod. Phys. 76, 1267-1305 (2005).

[6] John S. Bell, "On the Einstein Podolsky Rosen paradox," Physics Physique Fizika 1, 195-200 (1964).

[7] John S. Bell, "On the problem of hidden variables in quantum mechanics," Rev. Mod. Phys. 38, 447-452 (1966).

[8] Simon Kochen and E. P. Specker, "The Problem of Hidden Variables in Quantum Mechanics," Journal of Mathematics and Mechanics 17, 59-87 (1967).

[9] Lucien Hardy, "Quantum mechanics, local realistic theories, and Lorentz-invariant realistic theories," Phys. Rev. Lett. 68, 2981-2984 (1992).

[10] Borivoje Dakic and Caslav Brukner, "Quantum theory and beyond: Is entanglement special?" in Deep Beauty: Understanding the Quantum World through Mathematical Innovation (Cambridge University Press, 2011) pp. 365-392.

[11] Giulio Chiribella, Giacomo Mauro D'Ariano, and Paolo Perinotti, "Informational derivation of quantum theory," Phys. Rev. A 84, 012311 (2011).

[12] Rob Clifton, Jeffrey Bub, and Hans Halvorson, "Characterizing quantum theory in terms of information-theoretic constraints," Foundations of Physics 33, 1561-1591 (2003).

[13] Charles H. Bennett and Gilles Brassard, "Quantum cryptography: Public key distribution and coin tossing," Theoretical Computer Science 560, 7-11 (2014).

[14] Charles T. Sebens and Sean M. Carroll, "Self-locating Uncertainty and the Origin of Proba- 
bility in Everettian Quantum Mechanics," The British Journal for the Philosophy of Science 69, 25-74 (2016). 